Tohoku J. Exp. Med., 2013, 231, 293-298

\title{
Differential Expression of GAP-43 Protein in the Rostral Brain Neurons of Early Chick Embryos
}

\author{
Nozomi Onodera, ${ }^{1,2, *}$ Akinobu Kakehata ${ }^{1, *}$ and Isato Araki ${ }^{1,2}$ \\ ${ }^{1}$ Department of Chemistry and Bioengineering, Faculty of Engineering, Iwate University, Morioka, Iwate, Japan \\ ${ }^{2}$ The United Graduate School of Agricultural Sciences, Iwate University, Morioka, Iwate, Japan
}

\begin{abstract}
Brain development is composed of several processes, which are chronologically and mechanistically overlapping each other. However, the process of the earliest neural circuit formation in the rostral brain is less understood compared with other processes in brain development, in part because of the lack of appropriate molecular markers. Accordingly, the identification of molecular markers for nerve cells may accelerate the detailed analysis of neural development. Growth associated protein 43 (GAP-43) is a major growth cone protein that regulates F-actin dynamics, and it has been often used as a marker for developing neurons. To test whether GAP-43 can be used as a general marker for developing neurons in chick early embryos, we analyzed the expression pattern of GAP-43 protein in the brain. While the majority of the neurons were GAP-43 positive, the earliest neurons in the dorsal mesencephalon (future tectum) were GAP-43 negative. However, a subset of the GAP-43 negative neurons became positive at later stages. Such a difference in the expression of GAP-43 protein may contribute to the precise patterning of the neural circuits in the mesencephalon in the subsequent development. The earliest neurons in the telencephalon, which belong to the terminal nerve (TN), were also GAP-43 positive. Since the development of TN is poorly understood compared to other cranial nerves, GAP-43 could help the detailed analysis of the development of TN as the marker.
\end{abstract}

Keywords: chick embryo; descending tract of the mesencephalic nucleus of the trigeminus; growth associated protein 43; pioneer neuron; terminal nerve

Tohoku J. Exp. Med., 2013 December, 231 (4), 293-298. C 2013 Tohoku University Medical Press

\section{Introduction}

Brain development is composed of several processes, which are chronologically and mechanistically overlapping each other, e.g. neural induction, regionalization, neurogenesis, layer formation and neural circuit formation (reviewed in Sanes et al. 2012). Most of the processes are extensively studied in several model organisms, including mouse and chick, two major model animals. In contrast, the process of the earliest neural circuit formation in the rostral brain in the amniotes is less understood compared with other processes.

Chick embryos are popular model system for the analysis of brain regionalization as well as the development of neural circuit (Araki and Nakamura 1999; Nakamura 2001). Recently, Ware and Schubert (2011) described the detailed analysis of the earliest neural circuit development in the rostral brain of the chick embryo; the earliest axons, the medial longitudinal fascicle (MLF) axons appear at stage HH12 (for staging, see Hamburger and Hamilton 1951) in the posterior lateral diencephalon, which is followed by the appearance of the descending tract of the mesencephalic nucleus of the trigeminus (DTmesV) axon in the dorsal mesencephalon and the tract of the postoptic commissure (TPOC) axons on the ventral anterior diencephalon close to the optic stalk at HH14 (Ware and Schubert 2011).

The identification of molecular markers for nerve cells has accelerated the detailed analysis of neural development. For example, neurofilament, $\beta$ III tubulin and acetylated $\alpha$-tubulin are often used for pan-neuronal markers; vesicular glutamate transporter for glutaminergic neurons; glutamate decarboxylase for GABAergic neurons; glycine transporter 2 for glycinergic neurons markers; tyrosine hydroxylase for dopaminergic neurons; serotonin transporter for serotogenic neurons; choline acetyltransferase for cholinergic neurons; dopamine $\beta$-hydroxylase for noradrenergic/adrenergic neurons; histidine decarboxylase for histaminergic neurons; glial fibrillary acidic protein for astrocytes; and myelin proteolipid protein (PLP)/DM-20 for mylenating oligodendrocytes.

Received May 13, 2013; revised and accepted November 25, 2013. Published online December 5, 2013; doi: 10.1620/tjem.231.293.

*These two authors contributed equally to this work.

Correspondence: Isato Araki, Department of Chemistry and Bioengineering, Faculty of Engineering, Iwate University, 4-3-5 Ueda, Morioka, Iwate 020-8551, Japan.

e-mail: iaraki@iwate-u.ac.jp 

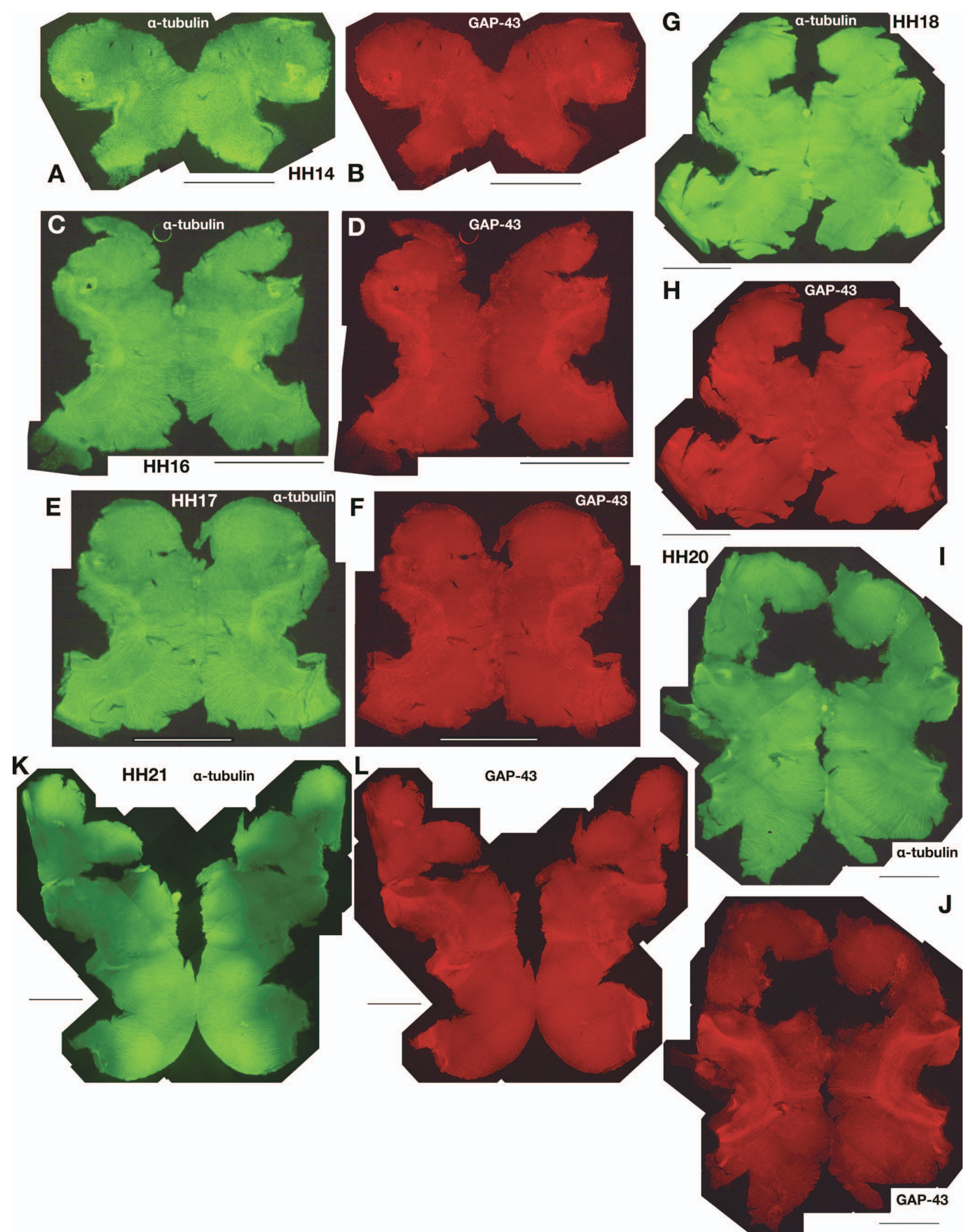

Fig. 1. Comparison of the expression patterns of acetylated $\alpha$-tubulin and GAP-43 in the rostral brain of chick embryo. Double immunofluorescence staining with anti-acetylated $\alpha$-tubulin antibody (in green; A, C, E, G, I, K) and antiGAP-43 antibody (in red; B, D, F, H, J, L). The stages are HH14 (A, B), HH16 (C, D), HH17 (E, F), HH18 (G, H), $\mathrm{HH} 20$ (I, J) and HH21 (K, L). The samples were flat-mounted so that the ventricular side faced to the glass slides after cutting along the ventral midline, coverslipped, and imaged through the coverslips. The pictures are schematized in 

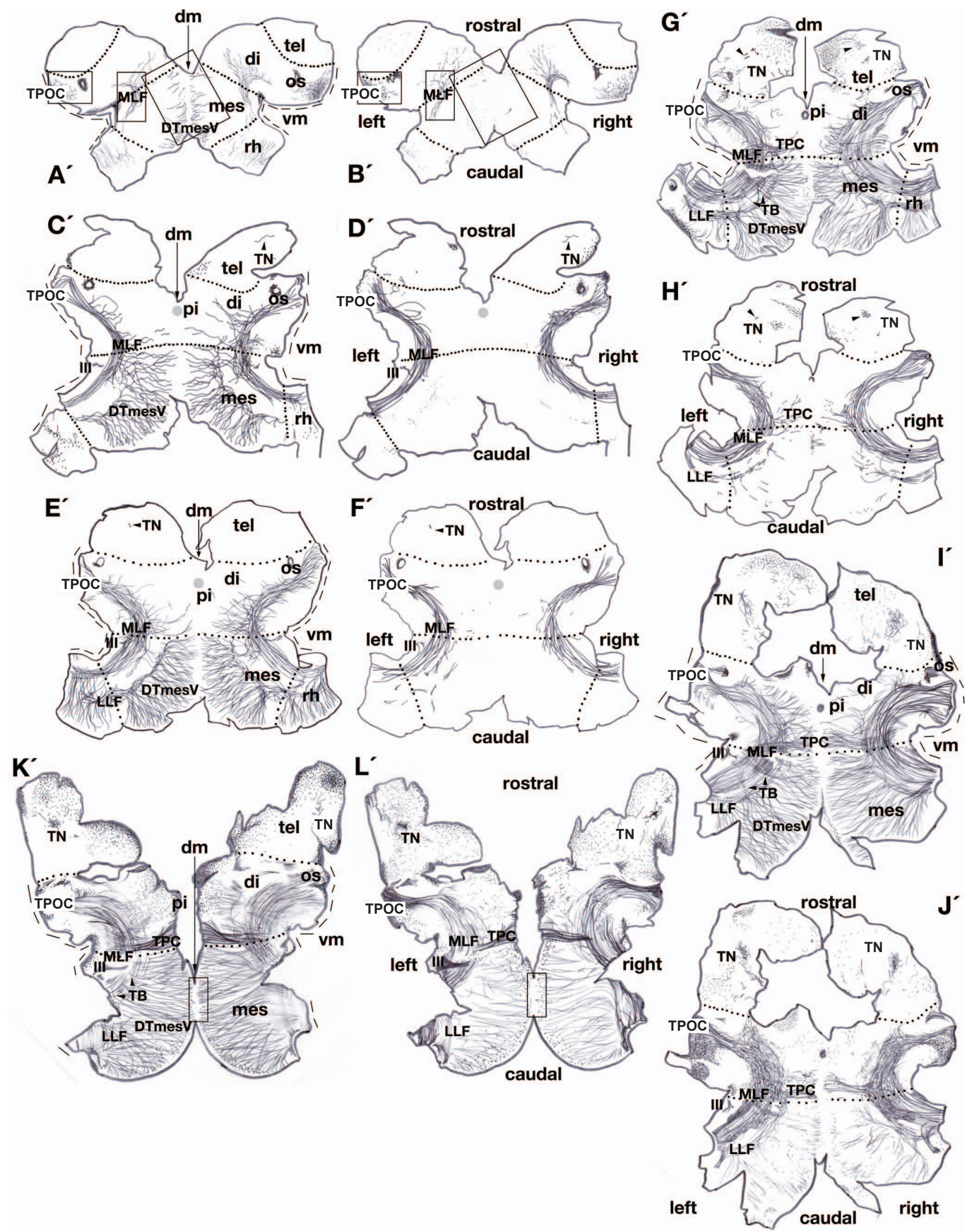

$\mathrm{A}^{\prime}-\mathrm{L}^{\prime}$. The dotted lines are the telencephalon-diencephalon, diencephalon-mesencephalon and mesencephalon-rhombencephalic boundaries. The dorsal midlines $(\mathrm{dm})$ are indicated with arrows. The broken lines indicate the ventral midlines (vm). Areas surrounded with rectangles are magnified in the Fig. 2. Abbreviations: di, diencephalon; III, oculomotor nerve; mes, mesencephalon; os, optic stalk; pi, pineal gland; rh, rhombencephalon; tel, telencephalon. For other abbreviations see the main text. Scale bars, $1 \mathrm{~mm}$. 
Growth associated protein 43 (GAP-43), also known as neuromodulin, B-50, pp46, brain abundant, membrane attached signal protein 2 (Basp2), P-57 and F1, consists of the loosely conserved IQ-motif, calmodulin-binding protein family with neurogranin (also known as RC3) and Purkinje cell protein 4 (also known as PEP-19) (for review, see Skene 1989; Benowitz and Routtenberg 1997). GAP-43 is a major growth cone protein, and regulates F-actin dynamics via its phosphorylation by protein kinase $\mathrm{C}$. It is also acylated at the N-terminal cysteine residues (Liang et al. 2002), and the N-terminal region is utilized to make a membrane-bound chimeric protein (Okada et al. 1999). Since the expression of GAP-43 mRNA and protein is almost confined to neurons, it is often used as a marker for developing neurons. However, GAP-43 is also expressed in glial cells and myoblasts (reviewed in Sensenbrenner et al. 1997).

Constitutive expression of GAP-43 in the neurons of transgenic mice induces sprouting of the axons in normal development as well as in the reinnervation after nerve lesion (Aigner et al. 1995). Although the adult central nervous system grossly normal in homozygous GAP-43 knockout mice, they show various defects in the neural circuits including failures of retinotectal projections, interhemispheric telencephalic connections and thalamocortical projections (Strittmatter et al. 1995; Maier et al. 1999; Zhang et al. 2000; Shen et al. 2002). At the cellular level the axon pathfinding is abnormal during the development and the growth cones are morphologically abnormal with reduced F-actin in vitro (Strittmatter et al. 1995; Shen et al. 2002). In addition the neuronal proliferation is also affected (Mani et al. 2001; Shen et al. 2008). Reflecting these structural defects GAP-43 knockout mice also show functional defects including memory impairment and sensorimotor disorder (Metz and Schwab 2004; Rekart et al. 2005).

To test whether GAP-43 can be used as a pan-neuronal marker in chick early embryos, we compared the immunofluorescence patterns between anti-GAP-43 polyclonal antibody and anti-acetylated $\alpha$-tubulin monoclonal antibody.

\section{Materials and Methods}

Embryos

Fertilized chicken eggs were purchased from a local farm. The eggs were incubated at $38^{\circ} \mathrm{C}$ until appropriate developmental stages (Hamburger and Hamilton 1951). The embryos were fixed with $4 \%$ paraformaldehyde in phosphate buffered saline (PBS) overnight, dehydrated in increasing concentrations of methanol and stored at $-20^{\circ} \mathrm{C}$ in $100 \%$ methanol until use. The study protocol was approved by the Animal Care and Use Committee of Iwate University. All experiments were performed in compliance with the relevant laws and the institutional guidelines.

\section{Immunofluorescence}

After rehydration through decreasing concentration of methanol the fixed embryos were incubated in blocking solution (5\% heat inactivated sheep serum) for $30 \mathrm{~min}$, and incubated with the primary antibodies, anti-GAP-43 rabbit polyclonal antibody (Novus Biologicals,
1:500 dilution in blocking solution) and anti-acetylated $\alpha$-tubulin monoclonal antibody (Sigma T6793, 1:4,000), overnight. Next, the embryos were washed in PBST (PBS with $0.1 \%$ Tween-20) for 10 min three time and for $1 \mathrm{hr}$ four times. Then, they were incubated in blocking solution for $30 \mathrm{~min}$, and incubated with secondary antibodies, Alexa Fluor 488 goat anti-mouse IgG $(\mathrm{H}+\mathrm{L})$ (Invitrogen, 1:500) and Alexa Fluor 594 goat anti-rabbit IgG $(\mathrm{H}+\mathrm{L})$ (Invitrogen, 1:500), overnight. Finally, they were washed with the same condition described above, flat-mounted by dissection, and observed with a conventional fluorescent microscope.

\section{Results and Discussion}

In the double immunostaining experiment with antiGAP-43 and anti-acetylated $\alpha$-tubulin antibodies we noticed that the MLF neurons/axons and TPOC neurons/axons are positive for GAP-43 while most of DTmesV neurons/axons are negative (Fig. 1A-C, A'-C'; Fig. 2A-F). This tendency was also observed at later stages (Fig. 1D-L, D'-L'; Fig. 2G and $\mathrm{H}$ ). Thus, the GAP-43 expression is not pan-neural in the brain of early chick embryos.

Previously, we showed that GAP-43 labeled the tract of the posterior commissure (TPC) in later embryos (HH23) (Matsunaga et al. 2000). TPC consists of dorsally projecting axons from neurons located in the ventral pretectum, posteriormost diencephalon, and ventrally projecting axons from neurons located in the dorsal pretectum. The former axons develop earlier than the latter (Fig. 1G; Ware and Schubert 2011). At HH18 when these axons start to extend dorsally, they are GAP-43-positive, and the expression continues at later stages (Fig. 1G-L, G'-L'). Thus, the TPC is GAP-43-positive as soon as it is formed.

While the posterior commissure crosses the dorsal midline at the posterior diencephalon, the mesencephalon has another commissure, tectal commissure. We asked whether any signs of the onset of the development of the tectal commissure can be detected among the specimens. However, we could not find any obvious evidence that a subset of axons cross the dorsal midline in the mesencephalon at the stages examined (Fig. 1; Fig. 2G and H), although further analyses are needed before definite conclusions can be reached.

It has been reported that the terminal nerve (TN) axons, the first axons in the telencephalon appear at HH17 (Ware and Schubert 2011). We observed that the putative TN cells expressed GAP-43 while the surrounding other telencephalic neurons only weakly expressed GAP-43 or were negative for it (Fig. 2I and J). In addition, the occulomotor nerve, another cranial nerve, was GAP-43 positive (Fig. 1C-L, $\mathrm{C}^{\prime}-\mathrm{L}^{\prime}$ ).

DTmesV neurons emerge at the earliest stage of the development of the amniotes; thus, they could function as pioneer neurons and could be crucial for later development of brain neural circuit (Ware and Schubert 2011). Since GAP-43 is generally enriched in developing neurons, the lack of GAP-43 in the most of developing chick DTmesV neurons was unexpected. Although the function of GAP-43 


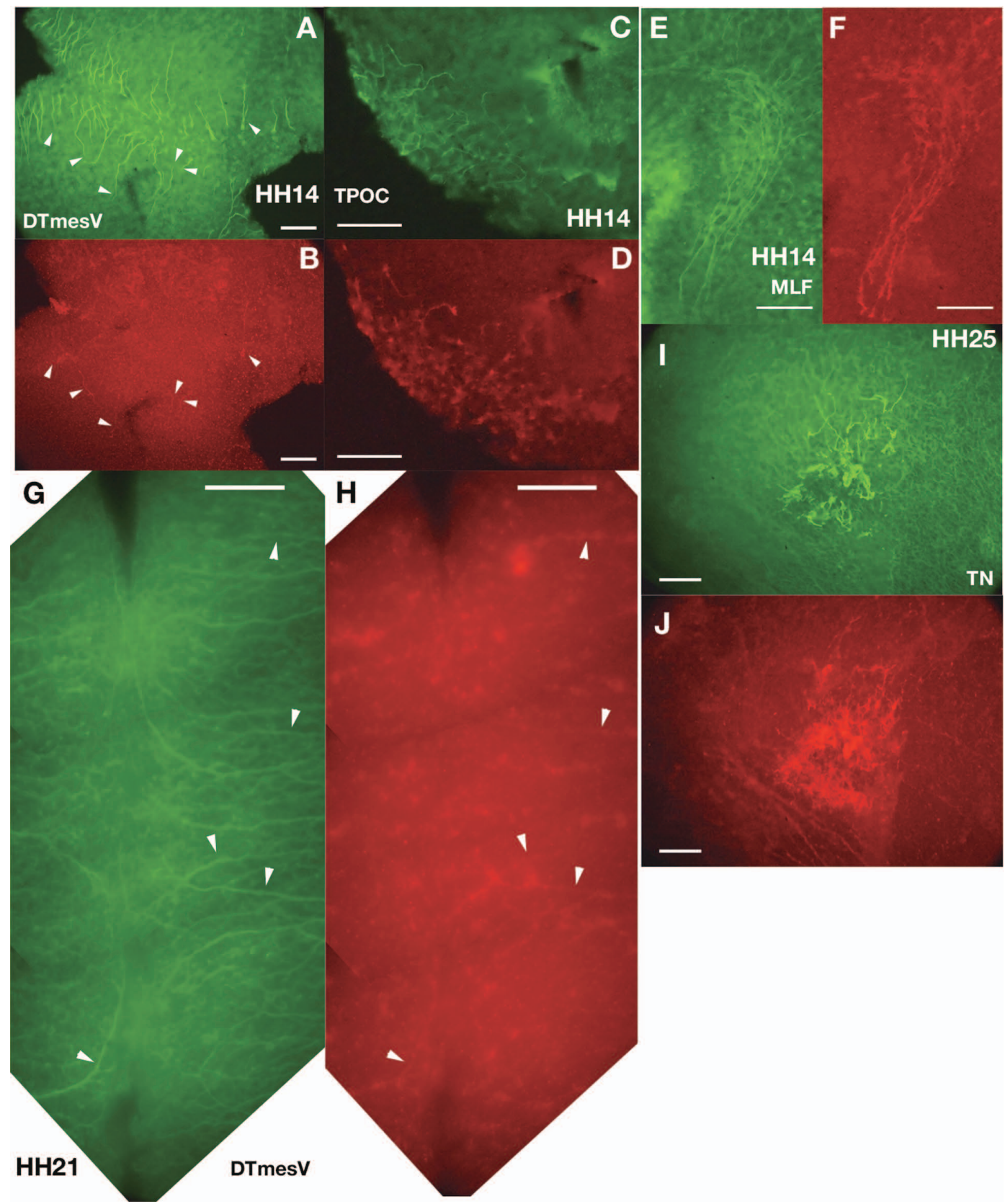

Fig. 2. Differential expression of GAP-43 protein in the chick developing axons.

Double immunofluorescence staining with anti-acetylated $\alpha$-tubulin antibody (in green; A, C, E, G, I) and anti-GAP-43 antibody (in red; B, D, F, H, J). A, B, C, D, E and F are higher magnification of the areas surrounded with rectangles in the Fig. $1 \mathrm{~A}^{\prime}$ and $\mathrm{B}^{\prime}$. G and $\mathrm{H}$ are higher magnification of the areas surrounded with rectangles in Fig. $1 \mathrm{~K}^{\prime}$ and $\mathrm{L}^{\prime}$. Most of DTmesV neurons are negative for GAP-43 (A, B) while TPOC (C, D) and MLF (E, F) neurons are positive at HH14, although small numbers of DTmesV neurons weakly expresses GAP-43 (arrowheads in A and B). The majority of DTmesV axons are still negative for GAP-43 at HH 21, while a subset of them are GAP-43 positive (G, H, arrowheads). (I, J) Putative TN cells are express GAP-43, while the expression in the surrounding telencephalic neurons is low or undetectable (HH25). Scale bars, $0.1 \mathrm{~mm}$.

seems to be critical for the development of neural circuits, and there is no structural paralogue with strong similarity in vertebrate genomes, a small number of homozygous GAP43-knockout mice survive even after birth (Strittmatter et al. 1995). This discrepancy is explained by the fact that a functional homologue of GAP-43 may be Basp1 (brain abundant, membrane attached signal protein 1/brain acid soluble protein 1), also known as CAP-23 (cytoskeletonassociated protein of approximate molecular mass $23 \mathrm{kD}$ ) and NAP-22 (22 kDa neuronal tissue-enriched acidic protein) (Frey et al. 2000). Thus, Basp1 might be expressed in chick DTmesV neurons and compensate for the lack of GAP-43 in the chick DTmesV neuron. Whether Basp1 is expressed in the DTmesV neurons or not, chick DTmesV 
neurons could be an ideal model system to assess the importance of GAP-43 during the development of neural circuits in amniotes in vivo.

Interestingly, we observed that a subset of axons in the dorsal/lateral mesencephalon were GAP-43 positive from stage 17 onward (Fig. 1E-L, E'-L'; Fig. 2A, B, G and H, arrowheads). The axonal trajectory indicates that at least some of the GAP-43 positive axons in the dorsal/lateral mesencephalon may be a subset of the DTmesV axons but not the tectobulbar (TB) axons, which originate from the lateral mesencephalon, cross the lateral longitudinal fascicle (LLF), the axonal bundle of DTmesV neurons, and join with the MLF. Thus, DTmesV neurons might be a heterogeneous population of neurons. Such heterogeneity might contribute to the precise patterning of the neural circuits in the mesencephalon at later stage. GAP-43 can be a useful molecular marker for the analysis of the heterogeneity in the DTmesV neurons. In addition, we detected the expression of GAP-43 in the developing putative TN cells, whose function is the least studied among cranial nerves. Thus, GAP-43 could be also used as a marker for TN cells, and facilitate the analysis of their development.

\section{Acknowledgements}

We would like to thank Prof. Harukazu Nakamura, Institute of Development, Aging and Cancer, Tohoku University for helpful comments. This work was supported by Iwate University Support Fund for Focused Research and for Formation of Center of Excellence.

\section{Conflict of Interest}

The authors declare no conflict of interest.

\section{References}

Aigner, L., Arber, S., Kapfhammer, J.P., Laux, T., Schneider, C., Botteri, F., Brenner, H.R. \& Caroni, P. (1995) Overexpression of the neural growth-associated protein GAP-43 induces nerve sprouting in the adult nervous system of transgenic mice. Cell, 83, 269-278.

Araki, I. \& Nakamura, H. (1999) Engrailed defines the position of dorsal di-mesencephalic boundary by repressing diencephalic fate. Development, 126, 5127-5135.

Benowitz, L.I. \& Routtenberg, A. (1997) GAP-43: an intrinsic determinant of neuronal development and plasticity. Trends Neurosci., 20, 84-91.

Frey, D., Laux, T., Xu, L., Schneider, C. \& Caroni, P. (2000) Shared and unique roles of CAP23 and GAP43 in actin regulation, neurite outgrowth, and anatomical plasticity. J. Cell Biol., 149, 1443-1454.

Hamburger, V. \& Hamilton, H.L. (1951) A series of normal stages in the development of the chick embryo. J. Morphol., 88, 49-92.

Liang, X., Lu, Y., Neubert, T.A. \& Resh, M.D. (2002) Mass spectrometric analysis of GAP-43/neuromodulin reveals the presence of a variety of fatty acylated species. J. Biol. Chem., 277, 33032-33040.

Maier, D.L., Mani, S., Donovan, S.L., Soppet, D., Tessarollo, L., McCasland, J.S. \& Meiri, K.F. (1999) Disrupted cortical map and absence of cortical barrels in growth-associated protein (GAP)-43 knockout mice. Proc. Natl. Acad. Sci. USA, 96, 9397-9402.

Mani, S., Shen, Y., Schaefer, J. \& Meiri, K.F. (2001) Failure to express GAP-43 during neurogenesis affects cell cycle regulation and differentiation of neural precursors and stimulates apoptosis of neurons. Mol. Cell. Neurosci., 17, 54-66.

Matsunaga, E., Araki, I. \& Nakamura, H. (2000) Pax6 defines the di-mesencephalic boundary by repressing En1 and Pax2. Development, 127, 2357-2365.

Metz, G.A. \& Schwab, M.E. (2004) Behavioral characterization in a comprehensive mouse test battery reveals motor and sensory impairments in growth-associated protein-43 null mutant mice. Neuroscience, 129, 563-574.

Nakamura, H. (2001) Regionalisation and acquisition of polarity in the optic tectum. Prog. Neurobiol., 65, 473-488.

Okada, A., Lansford, R., Weimann, J.M., Fraser, S.E. \& McConnell, S.K. (1999) Imaging cells in the developing nervous system with retrovirus expressing modified green fluorescent protein. Exp. Neurol., 156, 394-406.

Rekart, J.L., Meiri, K. \& Routtenberg, A. (2005) Hippocampaldependent memory is impaired in heterozygous GAP-43 knockout mice. Hippocampus, 15, 1-7.

Sanes, D.H., Reh, T.A. \& Harris, W.A. (2012) Development of the nervous system, 3rd ed., Academic Press, Burlington, MA.

Sensenbrenner, M., Lucas, M. \& Deloulme, J.C. (1997) Expression of two neuronal markers, growth-associated protein 43 and neuron-specific enolase, in rat glial cells. J. Mol. Med. (Berl.), 75, 653-663.

Shen, Y., Mani, S., Donovan, S.L., Schwob, J.E. \& Meiri, K.F. (2002) Growth-associated protein-43 is required for commissural axon guidance in the developing vertebrate nervous system. J. Neurosci., 22, 239-247.

Shen, Y., Mishra, R., Mani, S. \& Meiri, K.F. (2008) Both cellautonomous and cell non-autonomous functions of GAP-43 are required for normal patterning of the cerebellum in vivo. Cerebellum, 7, 451-466.

Skene, J.H. (1989) Axonal growth-associated proteins. Annu. Rev. Neurosci., 12, 127-156.

Strittmatter, S.M., Fankhauser, C., Huang, P.L., Mashimo, H. \& Fishman, M.C. (1995) Neuronal pathfinding is abnormal in mice lacking the neuronal growth cone protein GAP-43. Cell, 80, 445-452.

Ware, M. \& Schubert, F.R. (2011) Development of the early axon scaffold in the rostral brain of the chick embryo. J. Anat., 219, 203-216.

Zhang, F., Lu, C., Severin, C. \& Sretavan, D.W. (2000) GAP-43 mediates retinal axon interaction with lateral diencephalon cells during optic tract formation. Development, 127, 969980 . 